\title{
4th dimensional tracking: the GigaTracker of the NA62 experiment.
}

\section{E. Cortina Gil, E. Minucci, M. Perrin-Terrin; B. Velghe ${ }^{\dagger}$}

Université Catholique de Louvain, Belgium

S. Chiozzi, A. Cotta Ramusino, M. Fiorini, A. Gianoli, F. Petrucci, H. Wahl

INFN Sezione di Ferrara and University of Ferrara, Italy

\section{R. Arcidiacono $\ddagger$ C. Biino, F. Marchetto, E. Migliore}

INFN Sezione di Torino and University of Torino, Italy

G. Aglieri Rinella, D. Alvarez Feito, S. Bonacini, A. Ceccucci, J. Degrange, E. Gamberini, J. Kaplon, A. Kluge, A. Mapelli, M. Morel, J. Noël, M. Noy, L. Perktold, P. Petagna, K. Poltorak, G. Romagnoli, G. Ruggiero"

CERN, Geneva, Switzerland

\begin{abstract}
The GigaTracker is a lightweight hybrid silicon pixel detector built for the NA62 experiment at CERN, which aims at measuring the branching fraction of the ultra-rare kaon decay $K^{+} \rightarrow \pi^{+} v \bar{v}$ at the CERN SPS. The detector tracks charged particles in a $75 \mathrm{GeV} / c$ hadron beam with a flux reaching $1.3 \mathrm{MHz} / \mathrm{mm}^{2}$. It consists of three stations, $61 \times 27 \mathrm{~mm}^{2}$ each, which provide singlehit timing with $130 \mathrm{ps}$ resolution. Each station is composed of a $200 \mu \mathrm{m}$ thick planar silicon sensor, segmented in $300 \times 300 \mu \mathrm{m}^{2}$ pixels, bump-bonded to $2 \times 5$ custom $100 \mu \mathrm{m}$ thick ASIC, called TDCPix. Each TDCPix contains $40 \times 45$ asynchronous pixels, and is instrumented with 360 pairs of time-to-digital converter channels with 100 ps bin. The three stations are installed in vacuum (about $10^{-6}$ mbar) and cooled with liquid $\mathrm{C}_{6} \mathrm{~F}_{14}$ circulating through micro-channels etched inside silicon plates a few hundred microns thick. The total material budget is less than $0.5 \% X_{0}$ per station. Detector description, operational experience and performance from the NA62 experimental run in 2016 , at about $30 \%$ the nominal beam intensity, are presented.
\end{abstract}

The 26th International Workshop on Vertex Detectors

10-15 September, 2017

Las Caldas, Asturias, Spain

\footnotetext{
${ }^{*}$ Now at Aix Marseille Université, CNRS/IN2P3, CPPM, Marseille, France

${ }^{\dagger}$ Now at TRIUMF, Vancouver, Canada

$¥$ Also at Università degli Studi del Piemonte Orientale, Vercelli, Italy

${ }^{\S}$ Speaker.

ฯCorresponding author E-mail: ernesto.migliore@ unito.it

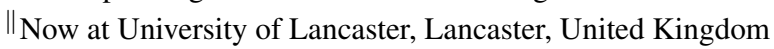




\section{The physics case for 4D tracking in the NA62 experiment}

NA62 is a fixed-target experiment at CERN SPS accelerator dedicated to the measurement of rare kaon decays $[1,2]$. In particular the experiment aims to measure the branching ratio of the ultra-rare decay $K^{+} \rightarrow \pi^{+} v \bar{v}$ at $10 \%$ precision, comparable with the current uncertainty of the Standard Model $(\mathrm{SM})$ prediction $B R=(8.4 \pm 1.0) \times 10^{-11}$ [3]. Assuming the SM branching ratio and a signal acceptance of $10 \%$, a sample of $10^{13} K^{+}$decays is required to collect $100 K^{+} \rightarrow \pi^{+} v \bar{v}$ events.

The experiment makes use of the technique of in-flight kaon decays: the large sample of kaons required for the measurement is obtained from a high intensity beam of unseparated secondary hadrons (K12 beam) produced impinging the $400 \mathrm{GeV} / c$ proton beam from the CERN SPS to a $400 \mathrm{~mm}$ long, $2 \mathrm{~mm}$ diameter beryllium target. The central momentum of the K12 beam is $75 \mathrm{GeV} / c$ with a r.m.s. $\Delta p / p=1 \%$. The fraction of $K^{+}$in the $\mathrm{K} 12$ beam is only $6 \%$, most of the particles being $\pi^{+}(70 \%)$ or protons (24\%).

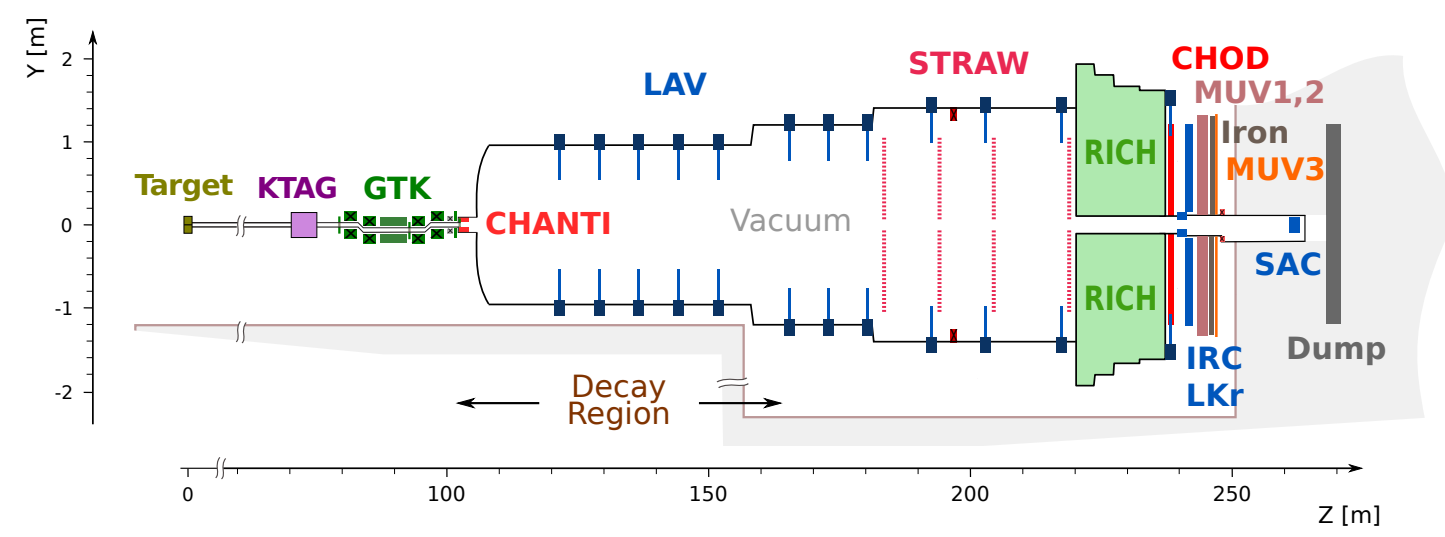

Figure 1: Schematic vertical section through the NA62 experimental setup. Useful $K^{+}$decays are detected in a $65 \mathrm{~m}$ long decay region after the GigaTracker. The main elements for the detection of the $K^{+}$decay products are located along a $150 \mathrm{~m}$ long region starting at $121 \mathrm{~m}$ downstream of the kaon production target. An evacuated passage surrounding the beam trajectory allows the intense $(750 \mathrm{MHz})$ flux of un-decayed beam particles to pass through without interacting with the detector material before reaching the dump.

Figure 1 shows a sketch of the NA62 detector: the kaons are identified by the KTAG [4], a differential Cherenkov counter located $70 \mathrm{~m}$ downstream from the target, and their momentum and direction are precisely measured by the three stations of the GigaTracker [5], while the tracks of the charged particles from the kaon decays are reconstructed by a four-chamber STRAW [6, 7] spectrometer. A $K^{+} \rightarrow \pi^{+} v \bar{v}$ event consists of a vertex between a kaon track measured by the GigaTracker and a single-detected pion track measured by the STRAW. The most abundant decay modes with a similar topology, $K^{+} \rightarrow \pi^{+} \pi^{0}(\gamma)$ and $K^{+} \rightarrow \mu^{+} v_{\mu}(\gamma)$, are rejected by a factor of $\mathscr{O}\left(10^{4}-10^{5}\right)$ selecting two restricted regions of the squared missing mass defined as:

$$
m_{m i s s}^{2}=\left(P_{K}-P_{\pi}\right)^{2}
$$

with $P_{K}\left(P_{\pi}\right)$ being the four-momentum of the parent (decay) particle assumed to be a kaon (charged pion). 
Differently from the LHC, the beam is not bunched but delivered in spills of $4.8 \mathrm{~s}$ (effective duration $3 \mathrm{~s}$ ): precise time measurement is therefore required to suppress combinatorics from particles in the initial and in the final state. To deal with a nominal intensity of the K12 beam of $750 \mathrm{MHz}$, the KTAG must provide measurements with 100 ps timing resolution. This sets the scale of the accuracy which has to be provided by the GigaTracker too. In that respect tracks reconstructed by the GigaTracker are truly four-dimensional as the time information of the hit is essential both for the pattern recognition phase and for the correct assignment of the track to the event.

\section{The GigaTracker detector}

The GigaTracker is a beam spectrometer which consists of three stations of hybrid pixel detectors and two pairs of dipole magnets, each pair arranged in an achromat configuration (Figure 2). The distance between the first two stations, GTK1 and GTK2, is $13.2 \mathrm{~m}$ and between the last two, GTK2 and GTK3, is $9.6 \mathrm{~m}$ with the last station located at $102.4 \mathrm{~m}$ from the target and marking the beginning of the fiducial decay region. A horizontal dipole (TRIM5) is placed $0.4 \mathrm{~m}$ in front of the last station to steer the beam, in combination with the magnet of the STRAW spectrometer, through the central aperture of the LKr calorimeter.
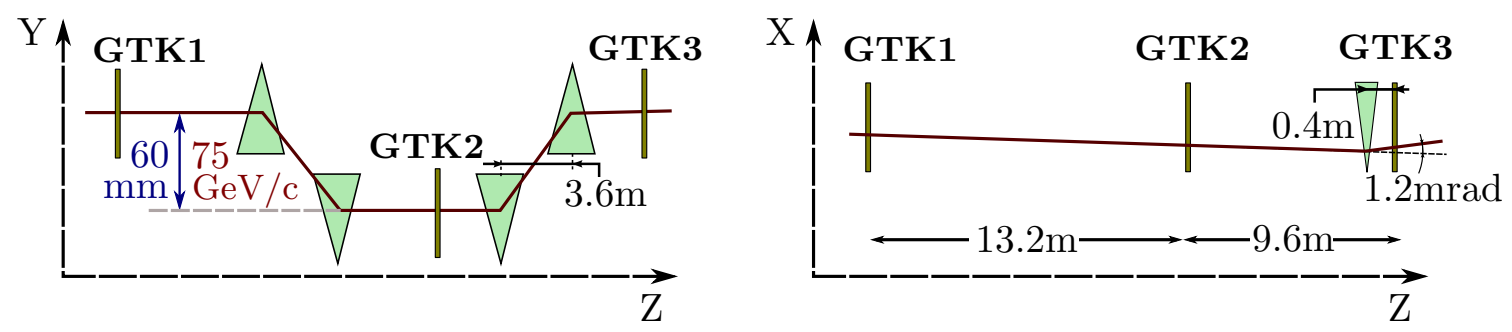

Figure 2: Schematic layout of the GigaTracker stations with the achromat and the TRIM5 magnets in the vertical and horizontal views respectively.

The design performances of the GigaTracker are:

- a momentum resolution of $0.2 \%$, with the momentum of the track measured by the vertical displacement of the track in the second station (about $60 \mathrm{~mm}$ for a $75 \mathrm{GeV} / \mathrm{c}$ momentum particle);

- a resolution on both directions ${ }^{1} \mathrm{dX} / \mathrm{dZ}$ and $\mathrm{dY} / \mathrm{dZ}$ of $16 \mu \mathrm{rad}$;

- a time resolution on a single hit of 200 ps leading to a 115 ps resolution for tracks with hits in the three stations.

These performances have to be met with a detector capable to sustain a high hit rate, up to $1.3 \mathrm{MHz} / \mathrm{mm}^{2}$ of charged hadrons in the region with the highest illumination, operating in a $10^{-6}$ mbar vacuum and providing the lowest amount of material budget $\left(0.5 \% X_{0}\right.$ per station, e.g. about $500 \mu \mathrm{m}$ of silicon). A GigaTracker station is shown in Figure 3. Each station consists of an assembly of:

\footnotetext{
${ }^{1}$ The NA62 experiment uses a right-handed reference system with the origin at the kaon production target, the $\mathrm{Z}$ axis defined by the beam line, the $\mathrm{Y}$ axis pointing vertically up, and the $\mathrm{X}$ axis horizontal and directed to form a right-handed coordinates system.
} 


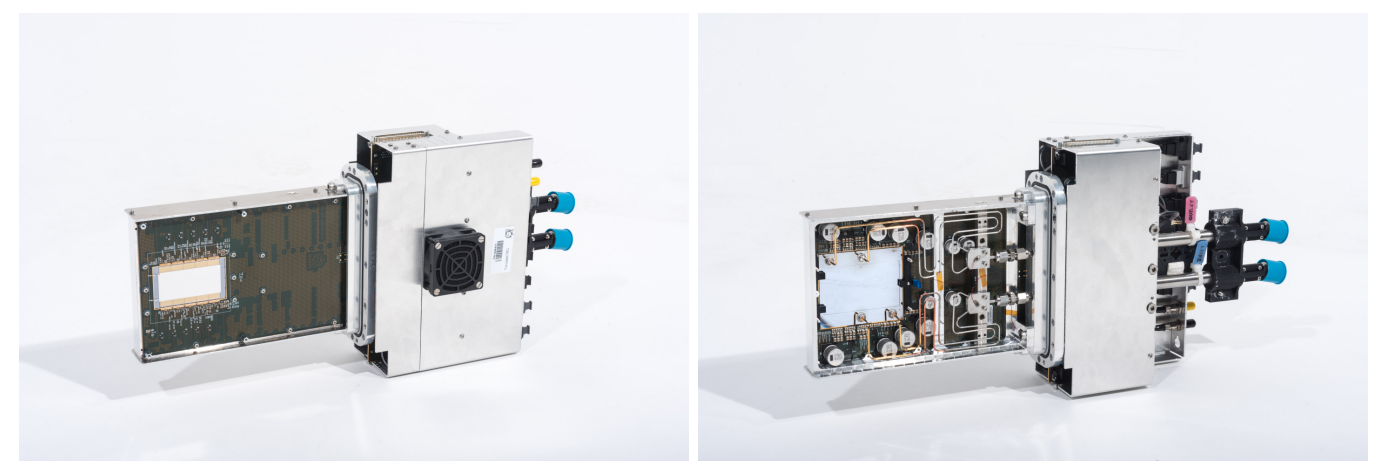

Figure 3: Picture of an assembled station looking at the sensor side (left) and at the cooling side (right). The beam enters in the detector from the cooling side.

- a $60.8(\mathrm{X}) \times 27.0(\mathrm{Y}) \mathrm{mm}^{2}$ silicon sensor segmented into $300 \times 300 \mu \mathrm{m}^{2}$ planar pixel cells for a total of 18000 pixels per station ${ }^{2}$; assemblies used in 2016 and 2017 data taking are made with $200 \mu \mathrm{m}$ thick $n$-on- $p$ sensors produced by FBK;

- ten TDCPix ASIC readout chips [8] arranged in two rows of five chips, built in CMOS $130 \mathrm{~nm}$ technology by IBM;

- a cooling plate with two groups of 75 micro-channels $\left(200 \times 70 \mu \mathrm{m}^{2}\right.$ cross-section) etched in a silicon substrate and containing the liquid $\mathrm{C}_{6} \mathrm{~F}_{14}$ coolant [9]. The design thickness of the cooling plate is $210 \mu \mathrm{m}$ although thicker plates are used in the current assemblies. The cooling plates are produced by CEA Leti.

The readout chips are connected to the sensors with SnAg bump bonds performed at IZM. IZM also performs the deposit of a $3 \mu \mathrm{m}$ thick layer of benzocyclobutene (BCB) to prevent sparks and the thinning of the ASIC down to $100 \mu \mathrm{m}$. The chips are then fixed to the cooling plate by a biadhesive tape and the assembly is clamped to a PCB card hosting the ancillary electronics. A steel vessel ending with a flange is used to mount the station in the secondary vacuum of the experiment. In case of need, the stations may be accessed and replaced during the routine interruptions of the operations of the SPS accelerator.

\section{The accurate measurement of the time of the hit}

The two keys to achieve the target timing measurement precision are a dedicated readout ASIC and a refined offline calibration procedure of the hit time.

\subsection{The TDCPix readout chip}

The TDCPix has two blocks:

- a matrix of $40 \times 45$ cells for the readout of the individual pixels; each cell features a preamplifier, a discriminator based on a 5-bit DAC and the corresponding section of the asynchronous transmission lines;

\footnotetext{
${ }^{2}$ The size along $\mathrm{X}$ of the pixels in the columns at the edges of neighbouring chips is $400 \mu \mathrm{m}$ to avoid interference between chips during the bump bonding step.
} 
- an end-of-column region hosting the TDCs and the digital logic.

The chip is organized by columns with one column made of 45 pixels. Groups of 5 pixels send signals passing the discriminator threshold to a hit arbiter which in turn sends the signal to a dual TDC recording the time of the leading and of the trailing edge. A DLL serving a pair of columns provides the fine timestamp with a $97.65 \mathrm{ps}$ bin size. Data are sent out by a $3.2 \mathrm{~Gb} / \mathrm{s}$ data transmitter serving a group of ten columns (quarter chip). The power consumption is $3.5 \mathrm{~W}$ per chip for a surface of about $2.5 \mathrm{~cm}^{2}$.

\subsection{The offline reconstruction of the hit time}

The time of a hit in a pixel is reconstructed offline by applying two corrections: a time offset correction and a time-walk correction.
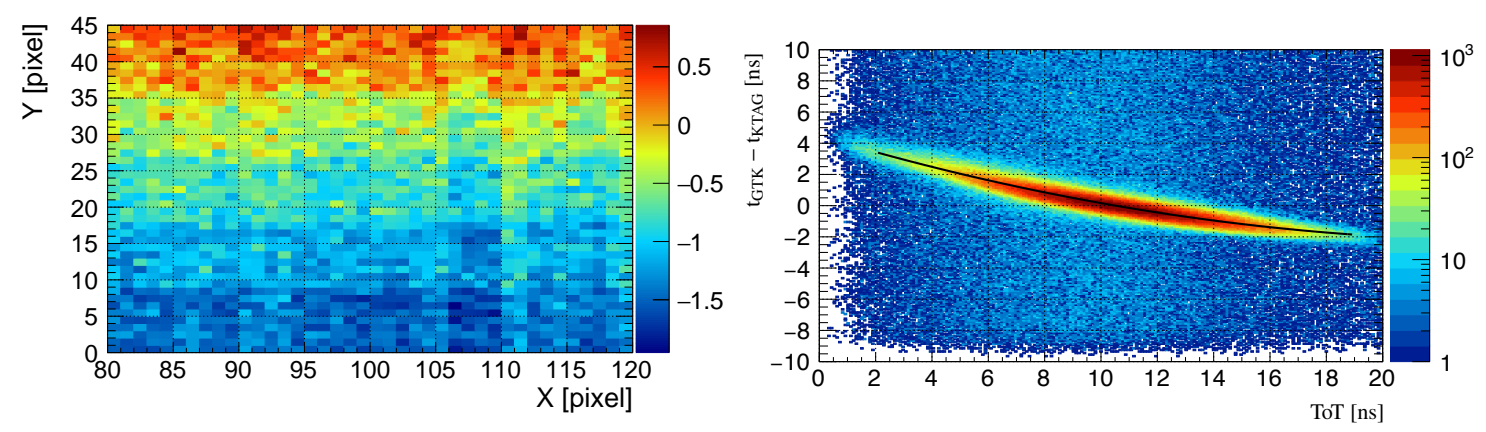

Figure 4: GigaTracker hit time corrections used in offline reconstruction. Left: time offsets in ns of the individual pixels in a TDCPix chip. Right: time-walk as a function of the signal height measured by the time-over-threshold (ToT). The line corresponds to the result of a quadratic fit of the most probable value of the time-walk.

The time offset corrections are determined for each chip on a run-by-run basis comparing the GigaTracker hit time to the time provided by other NA62 detectors like the KTAG. Final precision is achieved by computing a time offset which depends on the row and on the column of individual pixels. The offsets are recomputed every 100 runs, approximately one month of data taking. Figure 4 (left) shows a map of the time offsets computed for one chip: up to 2.5 ns difference is present between the first and the last pixels in a column.

The time-walk corrections take into account the Landau fluctuations of the signal, with larger signals crossing the discriminator threshold earlier than smaller signals. The need for time-walk corrections is apparent from Figure 4 (right) where the difference between the pixel hit time and the reference time from KTAG is shown as a function of the signal height measured by the timeover-threshold (ToT). At first order a $1 \mathrm{~ns}$ change in ToT corresponds to a $350 \mathrm{ps}$ shift of the hit time. The time-walk corrections are computed for each TDCPix chip and provided for the offline reconstruction in bins of ToT.

\section{Performance of the GigaTracker}

The GigaTracker is in its final configuration with the three stations fully operational since mid-September 2016. The same detector elements were used since then for the 2016 and 2017 data 


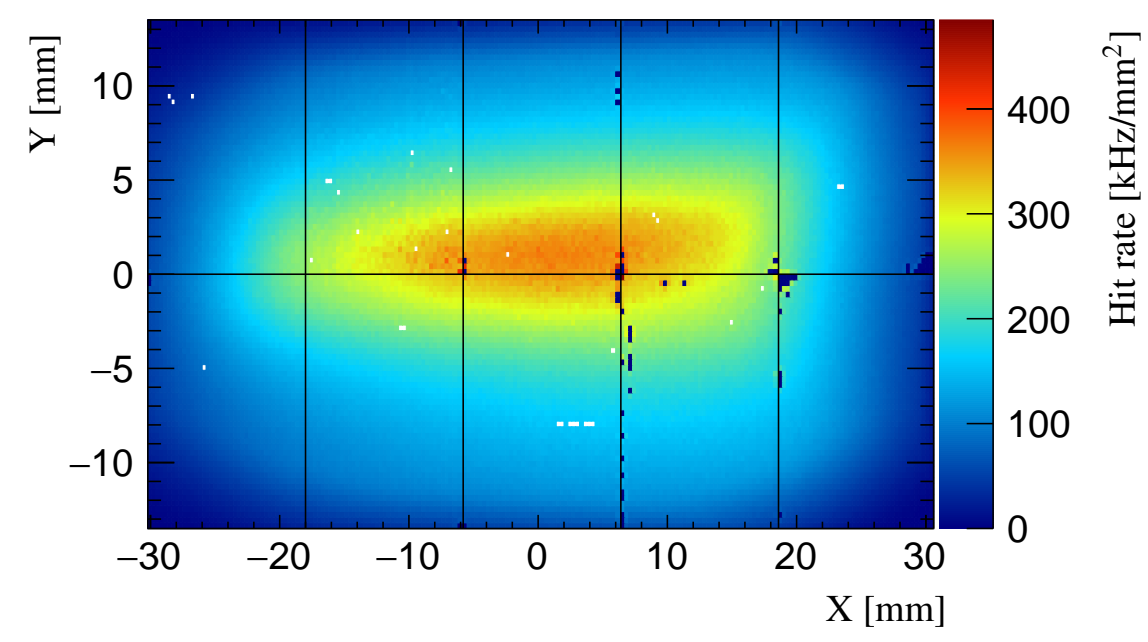

Figure 5: Hit rates in $\mathrm{kHz} / \mathrm{mm}^{2}$ in the GTK1 station operated in the beam at about $30 \%$ of the nominal intensity. The grid lines correspond to the chip boundaries.

taking $^{3}$ with a fraction of bad pixels at the level of few permill in all the stations. A hit map from a run taken at $30 \%$ of the nominal beam intensity, the typical beam conditions for the 2016 run, is shown in Figure 5. In 2017 the beam intensity raised up to $60 \%$ of the nominal value.

\subsection{Time resolution}

The time resolution in 2016 was evaluated using a sample of decays $K^{+} \rightarrow \pi^{+} \pi^{+} \pi^{-}$fully reconstructed by the STRAW spectrometer. The kaon candidates were then extrapolated to the GigaTracker and matched to a track reconstructed by the GigaTracker, with hits in all the three stations, using only geometrical and kinematic information. The differences of the hit times $t_{\mathrm{GTK} 1}-t_{\mathrm{GTK} 3}$, $t_{\mathrm{GTK} 2}-t_{\mathrm{GTK} 3}$ and $t_{\mathrm{GTK} 1}-t_{\mathrm{GTK} 2}$, are shown in Figure 6 . Assuming that the time measurements are not correlated, the hit time resolutions are $\sigma\left(t_{\mathrm{GTK} 1}\right)=132 \mathrm{ps}, \sigma\left(t_{\mathrm{GTK} 2}\right)=126 \mathrm{ps}$ and $\sigma\left(t_{\mathrm{GTK} 3}\right)=129 \mathrm{ps}$ resulting in a per-track time resolution of $74 \mathrm{ps}$. The expected contributions to the hit time resolution are: the TDCPix intrinsic resolution ( $80 \mathrm{ps})$, with this term including the finite size of the TDC bin and the jitter of the thresholds determining the time of the signal leading edge; the fluctuations of the signal shape due to the non uniformity of the energy deposit along the $200 \mu \mathrm{m}$ thickness of the sensor (100 ps); the non uniformity of the induced signal as a function of the hit position in the sensor plane, e.g. from the center of the cell to the edges. A test beam using a pixel telescope with high spatial resolution has been recently performed in order to precisely measure the different contributions to the time resolution. The analysis of the test beam data is underway.

Figure 7 shows the hit time resolution as a function of the bias voltage in a voltage scan performed at the end of 2016 data taking: the improvement when increasing the bias voltage from $100 \mathrm{~V}$, the standard operating conditions, to $250 \mathrm{~V}$ is about $15 \%$.

\footnotetext{
${ }^{3}$ During the 2016-2017 shutdown the assemblies were removed from the experiment and stored in cold.
} 

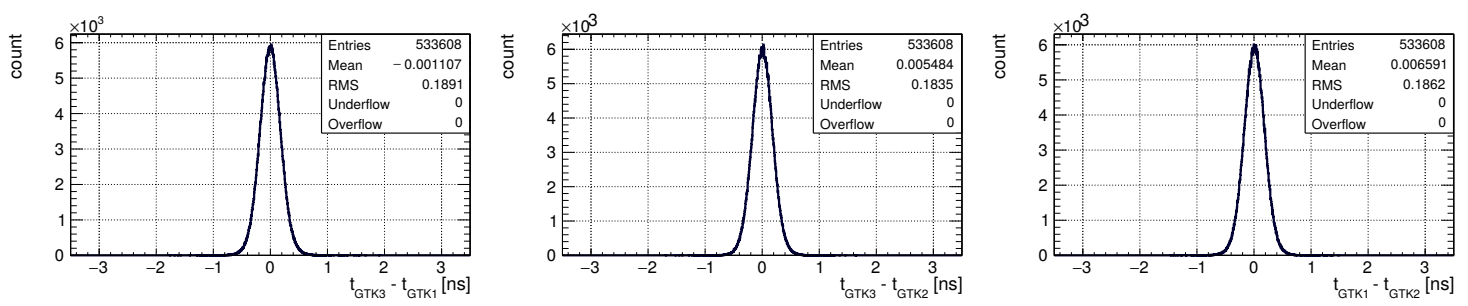

Figure 6: Time differences of pixel hits recorded in the three GigaTracker stations in fully reconstructed $K^{+} \rightarrow \pi^{+} \pi^{+} \pi^{-}$decays (2016 data). The RMS of the three distributions are: $R M S\left(t_{\mathrm{GTK} 1}-t_{\mathrm{GTK} 3}\right)=189 \mathrm{ps}$, $R M S\left(t_{\mathrm{GTK} 2}-t_{\mathrm{GTK} 3}\right)=184 \mathrm{ps}$ and $R M S\left(t_{\mathrm{GTK} 1}-t_{\mathrm{GTK} 2}\right)=186 \mathrm{ps}$. These values are only few ps larger than the standard deviations from a gaussian fit thus indicating the small contribution of non-gaussian tails.

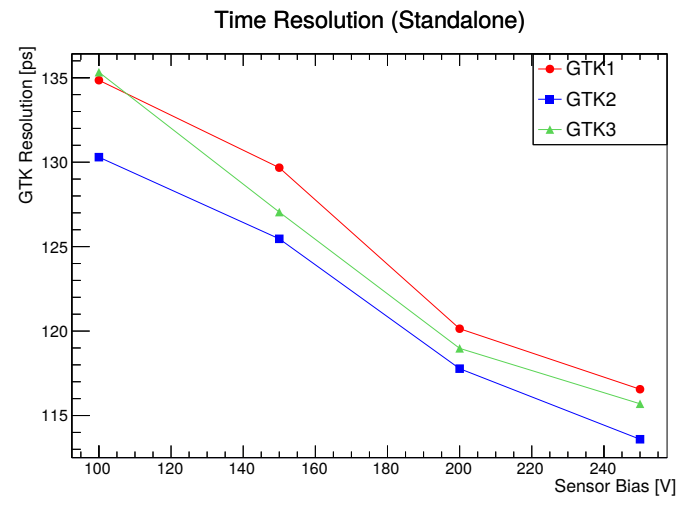

Figure 7: Time resolution as a functions of the bias voltage (2016 data). The standard operating conditions correspond to $100 \mathrm{~V}$.

\subsection{Spatial alignment}

The GigaTracker stations were aligned with respect to the STRAW spectrometer using fully reconstructed $K^{+} \rightarrow \pi^{+} \pi^{+} \pi^{-}$decays. The kaon candidates extrapolated to the GigaTracker were used both to determine the misalignment of the stations in the transverse plane and to equalize the measurement of the momentum done by the GigaTracker and by the STRAW spectrometer.

The performance of the spatial alignment was checked on a sample of fully reconstructed $K^{+} \rightarrow \pi^{+} \pi^{0}$ decays. The resolution on $m_{\text {miss }}^{2}$ as a function of the momentum of the charged pion is shown in Figure 8 [10]: the measured resolution agrees with the expectations for the design resolution of $16 \mu \mathrm{rad}$.

\subsection{Preview of the 2017 run}

The radiation at which the three assemblies used in 2016 and 2017 runs were exposed to is monitored with the counts recorded by a wire chamber located at the end of the beam line. On average, the estimated total ionizing dose (TID) received until the beginning of September 2017 was $7 \mathrm{kGy}$ and the non-ionizing energy loss (NIEL) was $1.5 \times 10^{13} 1 \mathrm{MeV} \mathrm{n}$ eq $/ \mathrm{cm}^{2}$. The peak values of TID and NIEL in the most illuminated area of the GigaTracker are approximately five times larger than the average and correspond to half a year of running the experiment at nominal beam intensity. 


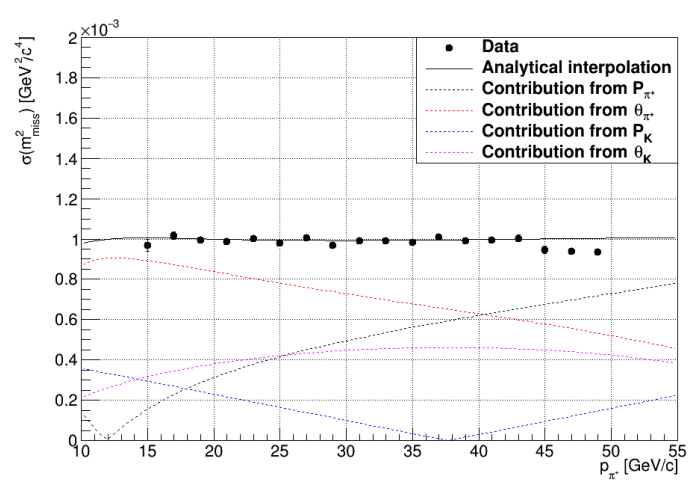

Figure 8: Gaussian resolution of the $m_{\text {miss }}^{2}$ measured on a $K^{+} \rightarrow \pi^{+} \pi^{0}$ sample as a function of charged pion momentum (2016 data). The black solid line is an analytical interpolation using the expected resolutions for GigaTracker and STRAW. The dashed lines correspond to the contributions of the individual quantities entering in the computation of $m_{m i s s}^{2}$.

In 2016 and 2017 the detector was run at cold temperature, in the range between $-10{ }^{\circ} \mathrm{C}$ and $-5{ }^{\circ} \mathrm{C}$, thus keeping the leakage current down to $10-15 \mu \mathrm{A}$ per station. The bias voltage used for 2017 operations was the same $(100 \mathrm{~V})$ used in 2016. Preliminary results indicate a small deterioration of the hit time resolution, about $140 \mathrm{ps,} \mathrm{which} \mathrm{is} \mathrm{anyway} \mathrm{largely} \mathrm{acceptable} \mathrm{for} \mathrm{carrying} \mathrm{on} \mathrm{the}$ physics program of the experiment. New assemblies are currently under production to replace the old ones for the future NA62 runs.

\section{Acknowledgments}

The cost of the GigaTracker development and construction were supported by the funding agencies of the GigaTracker group Institutes. We are particularly indebted to: F.R.S.-FNRS (Fonds de la Recherche Scientifique - FNRS), Belgium, INFN (Istituto Nazionale di Fisica Nucleare), Italy and CERN (European Organization for Nuclear Research), Switzerland. We are grateful to the whole NA62 Collaboration for operating the detector during data taking and providing the data used to evaluate the spectrometer performance.

\section{References}

[1] G. Anelli et al., Proposal to measure the rare decay $K^{+} \rightarrow \pi^{+} v \bar{v}$ at the CERN SPS, .

[2] NA62 collaboration, E. Cortina Gil et al., The beam and detector of the NA62 experiment at CERN, JINST 12 (2017) P05025, [1703.08501].

[3] A. J. Buras, D. Buttazzo, J. Girrbach-Noe and R. Knegjens, $K^{+} \rightarrow \pi^{+} v \bar{v}$ and $K_{L} \rightarrow \pi^{0} v \bar{v}$ in the Standard Model: status and perspectives, JHEP 11 (2015) 033, [1503.02693].

[4] E. Goudzovski et al., Development of the kaon tagging system for the NA62 experiment at CERN, Nucl. Instrum. Meth. A801 (2015) 86-94, [1509.03773].

[5] M. Fiorini et al., High rate particle tracking and ultra-fast timing with a thin hybrid silicon pixel detector, Nucl. Instrum. Meth. A718 (2013) 270-273. 
[6] NA62 collaboration, H. Danielsson, Performance and operation of the straw detector in the NA62 rare kaon decay experiment, in Proceedings, 2015 IEEE Nuclear Science Symposium and Medical Imaging Conference (NSS/MIC 2015): San Diego, California, United States, p. 7581950, 2016, DOI.

[7] S. Shkarovskiy, NA62 spectrometer to search for $K^{+} \rightarrow \pi^{+} v \bar{v}$, JINST 12 (2017) C02027.

[8] M. Noy et al., The front end electronics of the NA62 GigaTracker: Challenges, design and experimental measurements, Nucl. Phys. Proc. Suppl. 215 (2011) 198-200.

[9] G. Romagnoli, D. A. Feito, B. Brunel, A. Catinaccio, J. Degrange, A. Mapelli et al., Silicon micro-fluidic cooling for NA62 GTK pixel detectors, Microelectron. Eng. 145 (2015) 133-137.

[10] NA62 collaboration, 2017 NA62 Status Report to the CERN SPSC, CERN-SPSC-2017-013. SPSC-SR-208, CERN, Geneva, Mar. 2017. 